\title{
Research on Hospital Information Integration
}

\author{
Jinguo Wang ${ }^{1}$ and Na Wang ${ }^{2, a, *}$ \\ ${ }^{1}$ Department of Urology, First Hospital of Jilin University, Changchun, China \\ ${ }^{2}$ Department of Anesthesiology, First Hospital of Jilin University, Changchun, China \\ ${ }^{*}$ Corresponding author: Na Wang
}

\begin{abstract}
The traditional integration scheme has some disadvantages, such as complexity, high coupling degree of each system and large modification to the original system. The system integration platform is the combination of software and hardware, and it is the bridge and intermediary between various systems. Application system integration is realized through the integration platform, which forms a topology between the integration platform and each application system. Information interaction between systems is achieved through messaging. The medical supervision system should be set up. Development of a medical information management system includes integrated framework of medical security management system, dispute assessment system and protocol disposal system.
\end{abstract}

\section{Introduction}

After decades of development, domestic hospital information system has gradually established hospital management information system, laboratory management system, image archiving and communication system and radioactive information system [1]. However, most of these systems adopt different software manufacturers, apply different system architectures and different databases, and most of the information exchanges between these systems adopt the point-to-point interface pattern, one interface solves a problem, and the code cannot be reused, so the whole system becomes a complex network structure [2].

At the same time, the number of medical information subsystems is also increasing with the development of hospital information integration, and the complexity of connecting subsystems in a heterogeneous way is also increasing, which leads to insufficient information sharing and exchange between systems, forming an information island and seriously hindering the development of hospital information integration [3].

\section{Scheme based on hospital information platform}

Traditional integration solutions include point-to-point integration, message broker-based integration, component-based integration and middleware-based integration [4]. They are in for a certain aspects of integration may be a good solution, such as two systems by visiting each other open exchange of information interface, which USES a point-to-point integration is a quick and investment cost little, and when to deal with integration of multiple systems, in the past would consider using message broker or middleware platform.

\section{Disadvantages of traditional integration}

\subsection{Concept}

The hospital information sharing and application integration platform is based on standards such as HL7 and refers to the technical framework and architecture to integrate all kinds of medical information, including image, text, video and test data, seamlessly linking each independent medical information subsystem [5]. The integration platform is based on an open architecture and has strong scalability. It supports custom adapters to connect information systems that do not support HL7 standard, thus realizing the complete integration of old and new hospital systems.

\subsection{Design of platform framework}

The application integration platform adopts the application adapter technology to realize the connection and integration between the data exchange standard interface and the information integration sharing platform and the application system through configuration [6]. Change the connection mode based on programming to realize rapid integration between systems. Based on ESB technology design, it supports data transformation between different data transmission protocols, eliminates technical differences between different applications, enables different application services to coordinate and

\footnotetext{
a Corresponding author: wangna080613@163.com
} 
operate, and realizes communication and integration between different services.

The system is based on the management and monitoring system of $\mathrm{B} / \mathrm{S}$ structure, JMX management protocol and Web service technology, making the management and monitoring not limited by the system and application environment [7]. It is confirmed that the application integration platform and data system logic can not rely on any specific application system, independent of the application system vendors and system characteristics. Realize multiple data collection methods, realize one time collection and sharing, and avoid repeated input. It can be used as an entrance and exit for cross-hospital data sharing and regional medical treatment to avoid duplicate construction of the system [8].

\subsection{Enterprise service bus (ESB) and Adaptor technology}

Through the ESB technology to realize the conversion between different communication protocols, through different types of adapter configuration realization of business collaboration and data sharing, and HL7 engine embedded in the integrated platform, has realized the database to the HL7 message, HL7 message to a database, database to database, database to heterogeneous applications such as file data sharing, interface integration and method integrated with enterprise application integration (EAI) technology and application of adapter (Adaptor) technology, as well as XML and Web service technology [9].

\subsection{Service-oriented construction}

Using service oriented architecture (service-oriented architecture, SOA) is the application of different functional units, through well-defined interfaces and contracts between services, interfacing with neutral way of definition. It should be independent of implementation services hardware platform, operating system and programming language, which make the building in a variety of services in the system can interact in the form of a unit [10].

\subsection{Data integration}

Data integration is the foundation of process integration and desktop integration. Data integration means that each hospital system can access the information needed in the other system. Data integration can basically avoid duplicate input information and reduce the chance of errors [11]. But data integration is difficult to guarantee data consistency. Therefore, the principle of controlled access should always be followed in the integration process, that is, the data generated by the system should be responsible by the system itself, and other systems are not allowed to modify at will, so as to avoid inconsistency of data.

\section{Countermeasures to improve the information integration}

\subsection{Establishing a long-term goal}

Electronic information engineering construction of hospital administration has brought unprecedented opportunities and challenges, changed the traditional management way. Management is becoming easier. It is adapted to the medical personnel at the grass-roots level. The process of modern management mode needs certain transition period to make up the lack of cohesion of the hospital and management, let employees participate in the management with enthusiasm and confidence [12]. Only when all staffs take initiative to participate in the process of hospital management and strengthen the sense of ownership, the quality of hospital management can be improved and the level of medical service can be promoted.

\subsection{Automated-assistant management}

Automation technology aided electronic information engineering management is mainly refers to office management, electronic information engineering design and production process, involving a lot of design, manufacturing process and all aspects of the documents and data information, sorting and analysis of the information work is very important, if simply rely on artificial management, hard to avoid can reduce the efficiency of electronic information engineering office management, at the same time, there will be some mistakes [13]. The application of automation technology in electronic information engineering can effectively manage the design process, documents and data information through various software, and input information into the automatic management software system, which can fully guarantee the office management efficiency of electronic information engineering and promote the continuous development of electronic information engineering.

\subsection{Computers-integrated manufacturing}

It is determined by the characteristics of electronic information engineering design itself, and then compare the complex design process, you need to link together a lot of design, but also need a lot of relevant personnel and technology to cooperate with each other, in order to ensure the design is very convenience, to give full play to computer integrated manufacturing advantages, on the whole optimization [14]. In the actual process, but also give full play to the advantages of automation, using automated implements the aided design, ensuring each link is more optimization, but it is worth attention to each link of the optimization, and can't achieve the overall optimization, this would require the use of computer integrated manufacturing each optimized link in together, realize the whole optimization. In this way, the quality and efficiency of the designed products can be improved to varying degrees in a short time. 


\section{Conclusion}

Under the background of information time, people have been used to the application of electronic information technology. Electronic information engineering technology improves the intelligence of people's life, provides great convenience for people's life, and also promotes the development of society. In a macroscopic sense, information engineering is an inevitable product of the development of information modernization, which should be connected with electronic technology and is an inevitable demand of the modernization of social economy. On the based of this, it is of practical significance to strengthen the research on modern electronic information engineering technology.

\section{References}

1. Castells M, Hall P. Technopoles of the World: The making of 21st century industrial complexes, 1994.

2. Michael Crang. Tracey Crosbie, Stephen Graham. Variable Geometries of Connection: Urban Digital Divides and the Uses of Information Technology. Urban Studies, 2006.

3. Manuel Castells. Globalisation, Networking, Urbanisation: Reflections on the Spatial Dynamics of the Information Age. Urban Studies, 2010.

4. Ingwersen P. Cognitive perspectives of information retrieval interaction: elements of a cognitive IR theory. Journal of Documentation, 1996.
5. Amanda Spink, Charles Cole. J. Human information behavior: Integrating diverse approaches and information use. Am. Soc. Inf. Sci, 2005 (1).

6. TD. Wilson. Human Information Behavior. Informing Science, 2000.

7. Graham S, Marvin S. Telecommunications and the City: Electronic Spaces, Urban Places, 1996.

8. Fei JCH, Ranis G. Development of the Labor Surplus Economy: Theory and Policy, 1964.

9. The Vanishing City. Anthony Pascal. Urban Studies, 1987.

10. Louis Wirth. Urbanism as a Way of Life. The American Journal of Sociology, 1938.

11. B. Dervin. Users as Research Inventions: How Research Categories perpetuate Inequities. Journal of Communication, 1989.

12. David F. Batten. Network Cities: Creative Urban Agglomerations for the 21st Century. Urban Studies, 1995.

13. Harris Peter R, Sillence Elizabeth, Briggs Pam. Perceived threat and corroboration: key factors that improve a predictive model of trust in internet-based health information and advice. Journal of medical Internet research, 2011.

14. Ghulam Akhmat, Yu Bochun. Rapidly Changing Dynamics of Urbanization in China; Escalating Regional Inequalities and Urban Management Problems. Journal of Sustainable Development, 2010. 